\title{
ON THE UNIFORM CONVERGENCE OF WEIGHTED TRIGONOMETRIC SERIES
}

\author{
BOGDAN SZAL \\ University of Zielona Góra \\ Faculty of Mathematics, Computer Science and Econometrics \\ 65-516 Zielona Góra, ul. Szafrana 4a, Poland \\ E-mail: B.Szal@wmie.uz.zgora.pl
}

\begin{abstract}
In the present paper we consider a new class of sequences called $G M(\beta, r)$, which is the generalization of a class defined by Tikhonov in [15]. We obtain sufficient and necessary conditions for uniform convergence of weighted trigonometric series with $(\beta, r)$-general monotone coefficients.
\end{abstract}

1. Introduction. Chaundy and Jolliffe [1] proved the following classical result (see also [19]).

THEOREM 1. Suppose that $b_{n} \geq b_{n+1}$ and $b_{n} \rightarrow 0$. Then a necessary and sufficient condition for the uniform convergence of the series

$$
\sum_{n=1}^{\infty} b_{n} \sin n x
$$

is $n b_{n} \rightarrow 0$.

This result has been generalized by weakening the monotone conditions of the coefficient sequences. Generally speaking, it has become an important topic how to generalize monotonicity.

For $r \in \mathbb{N}$ and a sequence $\left(c_{n}\right)$, let

$$
\Delta_{r} c_{n}=c_{n}-c_{n+r} \quad \text { and } \quad \Delta c_{n}=\Delta_{1} c_{n} .
$$

2010 Mathematics Subject Classification : 40A30, 42A10.

Key words and phrases: Trigonometric series; uniform convergence; number sequences; best approximation.

The paper is in final form and no version of it will be published elsewhere. 
Recently, Leindler [4] defined a new class of sequences named as sequences of rest bounded variation, briefly denoted by $R B V S$, i.e.,

$$
R B V S=\left\{\left(c_{n}\right): \sum_{n=m}^{\infty}\left|\Delta c_{n}\right| \leq K(c)\left|c_{m}\right| \text { for all } m \in \mathbb{N}\right\},
$$

where here and throughout the paper $K(c)$ always denote a constant depending on indicated parameters, not necessarily the same in each occurrence.

Denote by $M S$ the class of monotone decreasing sequences and by $C Q M S$ the class of classic quasimonotone decreasing sequences ( $c \in C Q M S$ means that $c_{n} \in \mathbb{R}_{+}$for all $n \in \mathbb{N}$ and there exists an $\alpha>0$ such that $\left(c_{n} / n^{\alpha}\right)$ is decreasing $)$. It is obvious that

$$
M S \subset R B V S \cap C Q M S .
$$

Leindler [5] proved that the classes $C Q M S$ and $R B V S$ are not comparable. In [8] Leindler considered the class

$$
\begin{aligned}
& \operatorname{MRBVS}=\left\{\left(c_{n}\right): c_{n} \in \mathbb{C} \text { for all } n \in \mathbb{N}\right. \\
& \text { and } \left.\sum_{n=m}^{\infty}\left|\Delta c_{n}\right| \leq K(c) \frac{1}{m} \sum_{n \geq m / 2}^{m}\left|c_{n}\right| \text { for all } m \in \mathbb{N}\right\}
\end{aligned}
$$

of mean rest bounded variation sequences. Further, the class of general monotone coefficients, $G M$, is defined as follows (see [13]):

$$
G M=\left\{\left(c_{n}\right): c_{n} \in \mathbb{C} \text { for all } n \in \mathbb{N} \quad \text { and } \quad \sum_{n=m}^{2 m-1}\left|\Delta c_{n}\right| \leq K(c)\left|c_{m}\right| \text { for all } m \in \mathbb{N}\right\} .
$$

It is clear that

$$
R B V S \subset M R B V S \text { and } R B V S \cup C Q M S \subset G M .
$$

Very recently, Le and Zhou [3] suggested the following new class of sequences which includes $G M$ :

$$
\begin{aligned}
& G B V S=\left\{\left(c_{n}\right): c_{n} \in \mathbb{C} \text { for all } n \in \mathbb{N}\right. \\
& \text { and } \left.\sum_{n=m}^{2 m-1}\left|\Delta c_{n}\right| \leq K(c) \max _{m \leq n \leq N+m}\left|c_{n}\right| \text { for some integer } N \text { and all } m \in \mathbb{N}\right\} .
\end{aligned}
$$

The generalization of the Chaundy-Jolliffe criteria (Theorem 1) was studied in many papers: [9] for $C Q M S$, [4] for $R B V S$, [10] for $M R B V S$, [13] for $G M$ and [3] for $G B V S$.

In $[6,13,14,15]$ the class of $\beta$-general monotone sequences was examined as follows:

Definition 1. Let $\beta:=\left(\beta_{n}\right)$ be a nonnegative sequence. The sequence of complex numbers $c:=\left(c_{n}\right)$ is said to be $\beta$-general monotone, or $c \in G M(\beta)$, if the relation

$$
\sum_{n=m}^{2 m-1}\left|\Delta c_{n}\right| \leq K(c) \beta_{m}
$$

holds for all $m$. 
In [15] and [17] Theorem 1 was generalized to the class $G M\left(\beta^{*}\right)$, with

$$
\beta^{*}:=\left(\beta_{n}^{*}\right), \quad \beta_{n}^{*}=\sum_{k=[n / c]}^{[c n]} \frac{\left|c_{k}\right|}{k} \quad \text { for some } \quad c>1 .
$$

We can note that (see [15, Remark 2.1])

$$
G B V S \subset G M\left(\beta^{*}\right) .
$$

The next generalization of Theorem 1 was proved by Tikhonov and Dyachenko in [2]. They considered a class $G M\left(\beta^{\#}\right)$, with

$$
\beta^{\#}:=\left(\beta_{n}^{\#}\right), \quad \beta_{n}^{\#}=\frac{1}{n} \max _{k \geq[n / c]} \sum_{s=k}^{2 k}\left|c_{s}\right|
$$

and showed that

$$
G M\left(\beta^{*}\right) \subset G M\left(\beta^{\#}\right) .
$$

Moreover, they proved the following theorem.

THEOREM 2. (A) If

$$
\sum_{k=n}^{\infty}\left|\Delta b_{k}\right|=o\left(n^{-1}\right)
$$

as $n \rightarrow \infty$, then the series (1.1) converges uniformly.

(B) Let a nonnegative sequence $\left(b_{n}\right)$ satisfy

$$
b_{n} \leq K \cdot \frac{1}{n} \max _{k \geq[n / c]} \sum_{s=k}^{2 k} b_{s} \quad \text { for some } \quad c>1 .
$$

Then the uniform convergence of the series (1.1) implies $n b_{n}=o(1)$ as $n \rightarrow \infty$.

In order to formulate our new results we define another class of sequences (see [12]).

Definition 2. Let $\beta:=\left(\beta_{n}\right)$ be a nonnegative sequence and $r$ a natural number. The sequence of complex numbers $c:=\left(c_{n}\right)$ is said to be $(\beta, r)$-general monotone, or $c \in G M(\beta, r)$, if the relation

$$
\sum_{n=m}^{2 m-1}\left|\Delta_{r} c_{n}\right| \leq K(c) \beta_{m}
$$

holds for all $m$.

It is clear that $\operatorname{GM}(\beta, 1) \equiv G M(\beta)$. Moreover, the embedding relation between $G M(\beta, r)(r>1)$ and $G M(\beta, 1)$ can be deduced from the following remark:

REMARK $1([12])$. Let $r$ be a natural number. If a nonnegative sequence $\beta:=\left(\beta_{n}\right)$ is such that

$$
\sum_{i=0}^{r-1} \beta_{n+i} \leq K \cdot \beta_{n}
$$

for all $n$, then

$$
G M(\beta, 1) \subseteq G M(\beta, r)
$$


In [12] it was also showed that

$$
G M\left(\beta^{*}, 1\right) \subseteq G M\left(\beta^{*}, r\right) \quad \text { and } \quad G M\left(\beta^{\#}, 1\right) \subseteq G M\left(\beta^{\#}, r\right)
$$

for $r \geq 1$.

2. Statement of the results. We formulate our results in two subsections.

2.1. Uniform convergence of weighted trigonometric series. Let $r \in \mathbb{N}$ and $\alpha \in \mathbb{R}$. We define an even $2 \pi$-periodic function $\omega_{\alpha, r}$, given on the interval $[0, \pi]$ by the formula

$$
\omega_{\alpha, r}(x):= \begin{cases}\left(x-\frac{2 l \pi}{r}\right)^{\alpha} & \text { for } x \in\left(\frac{2 l \pi}{r}, \frac{(2 l+1) \pi}{r}\right] \text { and } l \in U_{1}, \\ \left(\frac{2(l+1) \pi}{r}-x\right)^{\alpha} & \text { for } x \in\left(\frac{(2 l+1) \pi}{r}, \frac{2(l+1) \pi}{r}\right) \text { and } l \in U_{2}, \\ 0 & \text { for } x=\frac{2 l \pi}{r} \text { and } l \in U_{3},\end{cases}
$$

where $U_{1}=\{0,1, \ldots,[r / 2]\}$ if $r$ is an odd number and $U_{1}=\{0,1, \ldots,[r / 2]-1\}$ if $r$ is even, $U_{2}=\{0,1, \ldots,[r / 2]-1\}$ for $r \geq 2$, and $U_{3}=\{0,1, \ldots,[r / 2]\}$ for $r \geq 1$.

Theorem 3. Let $r \in \mathbb{N}$ and $\alpha \in(0 ; 1]$. If

$$
\sum_{k=n}^{\infty}\left|\Delta_{r} b_{k}\right|=o\left(n^{\alpha-1}\right)
$$

as $n \rightarrow \infty$, then the series

$$
\sum_{k=1}^{\infty} b_{k} \omega_{\alpha, r}(x) \sin k x
$$

converges uniformly, and if

$$
\sum_{k=n}^{\infty}\left|\Delta_{r} a_{k}\right|=o\left(n^{\alpha-1}\right)
$$

as $n \rightarrow \infty$, then the series

$$
\sum_{k=1}^{\infty} a_{k} \omega_{\alpha, r}(x) \cos k x
$$

is also uniformly convergent.

THEOREM 4. Let $r=1$ or 2 and $\alpha \in(-1 ; 0]$. If (2.1) holds then the series (2.2) converges uniformly.

TheOREM 5. Let $r \in \mathbb{N}, \alpha \in \mathbb{R}$ and a nonnegative sequence $\left(b_{n}\right)$ satisfy

$$
b_{n} \leq K \sum_{k=[n / c]}^{[c n]} \frac{b_{k}}{k} \quad \text { for some } \quad c>1 .
$$

Then the uniform convergence of the series (2.2) implies

$$
n^{1-\alpha} b_{n}=o(1)
$$

as $n \rightarrow \infty$.

Similarly we can show the following theorem. 
TheOREM 5'. Let $r \in \mathbb{N}, \alpha \in \mathbb{R}$ and a nonnegative sequence $\left(a_{n}\right)$ satisfy

$$
a_{n} \leq K \sum_{k=[n / c]}^{[c n]} \frac{a_{k}}{k} \quad \text { for some } \quad c>1 .
$$

Then the uniform convergence of the series (2.3) implies

$$
n^{1-\alpha} a_{n}=o(1)
$$

as $n \rightarrow \infty$.

TheOREM 6. Let $r \in \mathbb{N}, \alpha \leq 0$ and a nonnegative sequence $\left(b_{n}\right)$ satisfy

$$
b_{n} \leq K \cdot \frac{1}{n} \max _{k \geq[n / c]} \sum_{s=k}^{2 k} b_{s} \quad \text { for some } \quad c>1 .
$$

Then the uniform convergence of the series (2.2) implies (2.5).

Proposition 1. (i) If a nonnegative sequence $\left(c_{n}\right) \in G M\left(\beta^{*}, r\right)$, where $r \in \mathbb{N}, \alpha<1$ and $n^{1-\alpha} c_{n}=o(1)$ as $n \rightarrow \infty$, then

as $n \rightarrow \infty$.

$$
n^{1-\alpha} \sum_{k=n}^{\infty}\left|\Delta_{r} c_{k}\right|=o(1)
$$

(ii) If a nonnegative sequence $\left(c_{n}\right) \in G M\left(\beta^{\#}, r\right)$, where $r \in \mathbb{N}, \alpha \leq 0$ and $n^{1-\alpha} c_{n}=$ $o(1)$ as $n \rightarrow \infty$, then (2.8) holds.

We conclude this subsection with a few remarks and corollary.

REMARK 2. If we take $r=1$ and $\alpha=0$ in Theorems 4 and 6 , then we obtain the result of M. Dyachenko and S. Tikhonov (Theorem 2).

Combining the above results we derive the following corollary.

Corollary 1. (i) Let $r \in \mathbb{N}, \alpha \in(0 ; 1)$ and a nonnegative sequence $\left(b_{n}\right) \in G M\left(\beta^{*}, r\right)$. Then the series (2.2) converges uniformly if and only if (2.5) holds.

(ii) Let $r \in \mathbb{N}, \alpha \in(0 ; 1)$ and a nonnegative sequence $\left(a_{n}\right) \in G M\left(\beta^{*}, r\right)$. Then the series (2.3) converges uniformly if and only if (2.6) holds.

(iii) Let $r=1$ or $2, \alpha \in(-1 ; 0]$ and a nonnegative sequence $\left(b_{n}\right) \in G M\left(\beta^{\#}, r\right)$. Then the series (2.2) converges uniformly if and only if (2.5) holds.

Remark 3. If we take $r=1$ and $\alpha=0$ in Corollary 1 (iii), then the result of M. Dyachenko and S. Tikhonov [2, Corollary 5.3] follows from our Corollary 1 (iii).

2.2. The weighted best approximation. Let $\gamma \in C$. Denote by $E_{n}(\varphi, \gamma)$ the best approximation of a function $\varphi$, where $\gamma \cdot \varphi \in C$, by trigonometric polynomials of degree at most $n$ in the weighted $C$-norm, that is,

$$
E_{n}(\varphi, \gamma):=\inf _{P_{n} \in \Pi_{n}}\left\|\gamma\left(\varphi-P_{n}\right)\right\| \quad\left(E_{n}(\varphi):=E_{n}(\varphi, 1)\right),
$$

where $\Pi_{n}$ denotes the set of all trigonometric polynomials of degree at most $n$.

Write

$$
f(x)=\sum_{k=1}^{\infty} a_{k} \cos k x, \quad g(x)=\sum_{k=1}^{\infty} b_{k} \sin k x
$$


for those $x$ where the series converge. Denote by $\phi$ either $f$ or $g$ and let $\lambda_{n}$ be its associated coefficients, i.e., $\lambda_{n}$ is either $a_{n}$ or $b_{n}$.

ThEOREM 7. Let $r \in \mathbb{N}, \alpha \in(0 ; 1]$. If

$$
\sum_{k=n}^{\infty}\left|\Delta_{r} \lambda_{k}\right|=o\left(n^{\alpha-1}\right)
$$

as $n \rightarrow \infty$, then

$$
E_{n}\left(\phi, \omega_{\alpha, r}\right) \leq K \max _{v \geq n}\left(v^{1-\alpha} \sum_{k=v}^{\infty}\left|\Delta_{r} \lambda_{k}\right|\right) .
$$

Analyzing the proofs of Theorem 4 and 7 we get the following corollary.

Corollary 2. If

$$
\sum_{k=n}^{\infty}\left|\Delta_{2} b_{k}\right|=o\left(n^{-1}\right)
$$

as $n \rightarrow \infty$, then

$$
E_{n}(g) \leq K \max _{v \geq n}\left(v \sum_{k=v}^{\infty}\left|\Delta_{2} \lambda_{k}\right|\right) .
$$

Using Proposition 1, Theorem 7 and Corollary 2 we can derive the next corollary.

Corollary 3. (i) Let $r \in \mathbb{N}, \alpha \in(0 ; 1)$ and a nonnegative sequence $\left(\lambda_{n}\right) \in G M\left(\beta^{*}, r\right)$. If $n^{1-\alpha} \lambda_{n}=o(1)$ as $n \rightarrow \infty$ then

$$
E_{n}\left(\phi, \omega_{\alpha, r}\right) \leq K \max _{v \geq n}\left(v^{1-\alpha} \lambda_{v}\right)
$$

(ii) If a nonnegative sequence $\left(b_{n}\right) \in G M\left(\beta^{\#}, 2\right)$ and $n b_{n}=o(1)$ as $n \rightarrow \infty$, then

$$
E_{n}(g) \leq K \max _{v \geq n}\left(v b_{v}\right) .
$$

Finally, we have the following remark.

REMARK 4. By the embedding relations (1.2) and (1.3) we can observe that the result of S. Tikhonov [15] follows from our Corollary 3 (ii).

3. Auxiliary results. Denote, for $r \in \mathbb{Z}$, by

$$
D_{k, r}(x)=\frac{\sin \left(k+\frac{r}{2}\right) x}{2 \sin \frac{r x}{2}}
$$

and

$$
\widetilde{D}_{k, r}(x)=\frac{\cos \left(k+\frac{r}{2}\right) x}{2 \sin \frac{r x}{2}}
$$

the Dirichlet type kernels.

LEMMA $1([11])$. Let $r \in \mathbb{N}, l \in \mathbb{Z}$ and $\left(c_{n}\right)$ be a sequence of complex numbers. If $x \neq \frac{2 l \pi}{r}$, then for all $m \geq n$

$$
\sum_{k=n}^{m} c_{k} \cos k x=\sum_{k=n}^{m} \Delta_{r} c_{k} D_{k, r}(x)-\sum_{k=m+1}^{m+r} c_{k} D_{k,-r}(x)+\sum_{k=n}^{n+r-1} c_{k} D_{k,-r}(x)
$$


and

$$
\sum_{k=n}^{m} c_{k} \sin k x=-\sum_{k=n}^{m} \Delta_{r} c_{k} \widetilde{D}_{k, r}(x)+\sum_{k=m+1}^{m+r} c_{k} \widetilde{D}_{k,-r}(x)-\sum_{k=n}^{n+r-1} c_{k} \widetilde{D}_{k,-r}(x) .
$$

\section{Proofs of the main results}

4.1. Proof of Theorem 3. We prove the theorem for sine series, only. In the case of cosine series we can argue analogously.

Let

$$
\varepsilon_{n}:=\sup _{v \geq n}\left(v^{1-\alpha} \sum_{k=v}^{\infty}\left|\Delta_{r} b_{k}\right|\right)
$$

and

$$
R_{n}(x):=\sum_{k=n}^{\infty} b_{k} \omega_{\alpha, r}(x) \sin k x .
$$

In view of the assumptions, we see that $\varepsilon_{n} \rightarrow 0$ as $n \rightarrow \infty$. Further, we shall show that

$$
\left|R_{n}(x)\right| \leq K \varepsilon_{n}
$$

for all $x \in \mathbb{R}$. Since $R_{n}\left(\frac{2 l \pi}{r}\right)=0$, where $l \in \mathbb{Z}$, it suffices to prove (4.1) for $x \in$ $\left(\frac{2 l \pi}{r} ; \frac{2(l+1) \pi}{r}\right)$, where $l=0,1, \ldots,[r / 2]-1$ if $r$ is even, and $l=0,1, \ldots,[r / 2]$ if $r$ is odd.

First, we show that (4.1) is valid for $x \in\left(\frac{2 l \pi}{r} ; \frac{2 l \pi}{r}+\frac{\pi}{r}\right]$. Let $N:=N(x)$ be the natural number such that

$$
\frac{2 l \pi}{r}+\frac{\pi}{N+1}<x \leq \frac{2 l \pi}{r}+\frac{\pi}{N} .
$$

Then

$$
R_{n}(x)=\sum_{k=n}^{n+N-1} b_{k} \omega_{\alpha, r}(x) \sin k x+\sum_{k=n+N}^{\infty} b_{k} \omega_{\alpha, r}(x) \sin k x=R_{n}^{(1)}(x)+R_{n}^{(2)}(x) .
$$

Further, by $(4.2)$ we obtain that for $\alpha \in(0 ; 1]$

$$
\begin{aligned}
\left|R_{n}^{(1)}(x)\right| \leq\left(x-\frac{2 l \pi}{r}\right)^{\alpha} \sum_{k=n}^{n+N-1} b_{k} \leq K N^{-\alpha} \sum_{k=n}^{n+N-1} \sum_{v=k}^{\infty}\left|\Delta_{r} b_{v}\right| \\
\leq K \varepsilon_{n} N^{-\alpha} \sum_{k=n}^{n+N-1} k^{\alpha-1} \leq K \varepsilon_{n} N^{-\alpha}\left((n+N)^{\alpha}-n^{\alpha}\right) \leq K \varepsilon_{n} .
\end{aligned}
$$

Using Lemma 1, the inequality

$$
\frac{r}{\pi} x-2 l \leq\left|\sin \frac{r x}{2}\right| \text { for } x \in\left[\frac{2 l \pi}{r}, \frac{2 l \pi}{r}+\frac{\pi}{r}\right]
$$


and (4.2) we get for $\alpha \leq 1$

$$
\begin{gathered}
\left|R_{n}^{(1)}(x)\right|=\left(x-\frac{2 l \pi}{r}\right)^{\alpha}\left|\sum_{k=n+N}^{n+N+r-1} b_{k} \widetilde{D}_{k,-r}(x)+\sum_{k=n+N}^{\infty} \Delta_{r} b_{k} \widetilde{D}_{k, r}(x)\right| \\
\leq K \frac{\left(x-\frac{2 l \pi}{r}\right)^{\alpha}}{2\left|\sin \frac{r x}{2}\right|}\left\{\sum_{k=n+N}^{\infty}\left|\Delta_{r} b_{k}\right|+\sum_{k=n+N}^{n+N+r-1} b_{k}\right\} \leq K\left(x-\frac{2 l \pi}{r}\right)^{\alpha-1} \sum_{k=n+N}^{\infty}\left|\Delta_{r} b_{k}\right| \\
\leq K N^{1-\alpha} \sum_{k=n+N}^{\infty}\left|\Delta_{r} b_{k}\right| \leq K \varepsilon_{n}\left(\frac{N}{n+N}\right)^{1-\alpha} \leq K \varepsilon_{n} .
\end{gathered}
$$

Now, we prove that $(4.1)$ is valid for $x \in\left[\frac{2 l \pi}{r}+\frac{\eta}{r} ; \frac{2(l+1) \pi}{r}\right)$. Let $M:=M(x) \geq r$ be the natural number such that

$$
\frac{2(l+1) \pi}{r}-\frac{\pi}{M} \leq x<\frac{2(l+1) \pi}{r}-\frac{\pi}{M+1} .
$$

Then

$$
R_{n}(x)=\sum_{k=n}^{n+M-1} b_{k} \omega_{\alpha, r}(x) \sin k x+\sum_{k=n+M}^{\infty} b_{k} \omega_{\alpha, r}(x) \sin k x=R_{n}^{(3)}(x)+R_{n}^{(4)}(x) .
$$

Using (4.2) we obtain that for $\alpha \in(0 ; 1]$

$$
\begin{aligned}
\left|R_{n}^{(4)}(x)\right| \leq\left(\frac{2(l+1) \pi}{r}-x\right) \sum_{k=n}^{\alpha} b_{k} \leq K M^{-\alpha} \sum_{k=n}^{n+M-1} \sum_{v=k}^{\infty}\left|\Delta_{r} b_{v}\right| \\
\leq K \varepsilon_{n} M^{-\alpha} \sum_{k=n}^{n+M-1} k^{\alpha-1} \leq K \varepsilon_{n} .
\end{aligned}
$$

Applying Lemma 1, the inequality

$$
2(l+1)-\frac{r}{\pi} x \leq\left|\sin \frac{r x}{2}\right| \text { for } x \in\left[\frac{2 l \pi}{r}+\frac{\pi}{r}, \frac{2(l+1) \pi}{r}\right]
$$

and (4.2) we get for $\alpha \leq 1$

$$
\begin{gathered}
\left|R_{n}^{(4)}(x)\right|=\left(\frac{2(l+1) \pi}{r}-x\right)^{\alpha}\left|\sum_{k=n+M}^{n+M+r-1} b_{k} \widetilde{D}_{k,-r}(x)+\sum_{k=n+M}^{\infty} \Delta_{r} b_{k} \widetilde{D}_{k, r}(x)\right| \\
\leq K \frac{\left(\frac{2(l+1) \pi}{r}-x\right)^{\alpha}}{2\left|\sin \frac{r x}{2}\right|}\left\{\sum_{k=n+M}^{\infty}\left|\Delta_{r} b_{k}\right|+\sum_{k=n+M}^{n+M+r-1} b_{k}\right\} \\
\leq K\left(\frac{2(l+1) \pi}{r}-x\right)^{\alpha-1} \sum_{k=n+M}^{\infty}\left|\Delta_{r} b_{k}\right| \\
\leq K M^{1-\alpha} \sum_{k=n+M}^{\infty}\left|\Delta_{r} b_{k}\right| \leq K \varepsilon_{n}\left(\frac{M}{n+M}\right)^{1-\alpha} \leq K \varepsilon_{n} .
\end{gathered}
$$

From the estimations (4.3), (4.4), (4.6), (4.8), (4.9), and (4.11) the uniform convergence of the series (2.2) follows and thus the proof is complete. 
4.2. Proof of Theorem 4. The proof of Theorem 4 goes analogously as the proof of Theorem 3. The only difference is that instead of (4.4) (for $r=1,2$ ) and (4.9) (for $r=2$ ) we have to use the following considerations.

Applying the inequalities $|\sin k x| \leq k x$ for $x \in(0, \pi),|\sin k x| \leq k(\pi-x)$ for $x \in(0, \pi)$ we obtain that for $\alpha \in(-1 ; 0]$

$$
\begin{aligned}
\left|R_{n}^{(1)}(x)\right| \leq x^{\alpha+1} & \sum_{k=n}^{n+N-1} k b_{k} \leq K N^{-\alpha-1} \sum_{k=n}^{n+N-1} \sum_{v=k}^{\infty}\left|\Delta_{r} b_{v}\right| \\
\leq K \varepsilon_{n} N^{-\alpha-1} & \sum_{k=n}^{n+N-1} k^{\alpha} \leq K \varepsilon_{n} N^{-\alpha-1}\left((n+N)^{\alpha+1}-n^{\alpha+1}\right) \leq K \varepsilon_{n}
\end{aligned}
$$

and

$$
\begin{aligned}
\left|R_{n}^{(4)}(x)\right| \leq(\pi-x)^{\alpha+1} \sum_{k=n}^{n+M-1} k b_{k} \leq K M^{-\alpha-1} & \sum_{k=n}^{n+M-1} \sum_{v=k}^{\infty}\left|\Delta_{r} b_{v}\right| \\
& \leq K \varepsilon_{n} M^{-\alpha-1} \sum_{k=n}^{n+M-1} k^{\alpha-1} \leq K \varepsilon_{n} .
\end{aligned}
$$

These estimates complete the proof.

4.3. Proof of Theorem 5. Suppose that the series (2.2) converges uniformly. Setting $x=\frac{\pi}{4 c m}$, where $c>1,4 c m \geq r$ and $r \in \mathbb{N}$, we get

$$
\sum_{n=[m / c]}^{[c m]} b_{n} \omega_{\alpha, r}(x) \sin n x=\left(\frac{\pi}{4 c m}\right)^{\alpha} \sum_{n=[m / c]}^{[c m]} b_{n} \sin \frac{n \pi}{4 c m} \geq K m^{-\alpha} \sum_{n=[m / c]}^{[c m]} b_{n} .
$$

Hence

$$
m^{-\alpha} \sum_{n=[m / c]}^{[c m]} b_{n}=o(1) \quad \text { as } \quad m \rightarrow \infty .
$$

If $\left(b_{n}\right)$ satisfies $(2.4)$ then

$$
n^{1-\alpha} b_{n} \leq K n^{1-\alpha} \sum_{k=[n / c]}^{[c n]} \frac{b_{k}}{k} \leq K n^{-\alpha} \sum_{k=[n / c]}^{[c n]} \frac{b_{k}}{k}
$$

and $n^{1-\alpha} b_{n}=o(1)$ as $n \rightarrow \infty$. This finishes the proof.

4.4. Proof of Theorem 6. Suppose that the series (2.2) converges uniformly. Setting $x=\frac{\pi}{4 m}$, where $4 m \geq r$ and $r \in \mathbb{N}$, we get

$$
\sum_{n=m}^{2 m} b_{n} \omega_{\alpha, r}(x) \sin n x=\left(\frac{\pi}{4 m}\right)^{\alpha} \sum_{n=m}^{2 m} b_{n} \sin \frac{n \pi}{4 m} \geq\left(\frac{\pi}{4}\right)^{\alpha} \sin \frac{\pi}{4} m^{-\alpha} \sum_{n=m}^{2 m} b_{n} .
$$

Hence

$$
m^{-\alpha} \sum_{n=m}^{2 m} b_{n}=o(1) \quad \text { as } \quad m \rightarrow \infty
$$


If $\left(b_{n}\right)$ satisfies (2.7), then for $c>1$ and $\alpha \leq 0$

$$
n^{1-\alpha} b_{n} \leq K n^{-\alpha} \max _{k \geq[n / c]}\left(\sum_{s=k}^{2 k} b_{s}\right) \leq K \max _{k \geq[n / c]}\left(k^{-\alpha} \sum_{s=k}^{2 k} b_{s}\right) .
$$

Therefore $n^{1-\alpha} b_{n}=o(1)$ as $n \rightarrow \infty$ and it completes the proof.

4.5. Proof of Proposition 1. (i) Let $n^{1-\alpha} c_{n}=o(1)$ as $n \rightarrow \infty$. Then

$$
n^{-\alpha} \sum_{k=[n / c]}^{[c n]} c_{k} \leq K \sum_{k=[n / c]}^{[c n]} k^{-\alpha} c_{k} \leq K \sup _{k \geq[n / c]}\left(k^{1-\alpha} c_{k}\right) \sum_{k=[n / c]}^{[c n]} \frac{1}{k} \leq K \sup _{k \geq[n / c]}\left(k^{1-\alpha} c_{k}\right) \text {. }
$$

Hence

$$
n^{-\alpha} \sum_{k=[n / c]}^{[c n]} c_{k}=o(1) \quad \text { as } \quad n \rightarrow \infty .
$$

Further, if $\left(c_{n}\right) \in G M\left(\beta^{*}, r\right)$, then for $\alpha<1$

$$
\begin{aligned}
n^{1-\alpha} \sum_{k=n}^{\infty}\left|\Delta_{r} c_{k}\right|= & n^{1-\alpha} \sum_{s=0}^{\infty} \sum_{k=2^{s} n}^{2^{s+1} n-1}\left|\Delta_{r} c_{k}\right| \leq K n^{1-\alpha} \sum_{s=0}^{\infty} \sum_{k=\left[2^{s} n / c\right]}^{\left[c 2^{s} n\right]} \frac{c_{k}}{k} \\
& \leq K n^{1-\alpha} \sum_{s=0}^{\infty} \frac{1}{\left(2^{s} n\right)^{1-\alpha}} \sup _{m \geq 2^{s} n}\left(m^{-\alpha} \sum_{k=[m / c]}^{[c m]} c_{k}\right) \\
& \leq K \sup _{m \geq n}\left(m^{-\alpha} \sum_{k=[m / c]}^{[c m]} c_{k}\right) \sum_{s=0}^{\infty}\left(\frac{1}{2^{1-\alpha}}\right)^{s} \leq K \sup _{m \geq n}\left(m^{-\alpha} \sum_{k=[m / c]}^{[c m]} c_{k}\right)
\end{aligned}
$$

and (2.8) holds.

(ii) Suppose $n^{1-\alpha} c_{n}=o(1)$ as $n \rightarrow \infty$. Then

$$
n^{-\alpha} \sum_{k=n}^{2 n} c_{k} \leq K \sup _{k \geq n}\left(k^{1-\alpha} c_{k}\right) \sum_{k=n}^{2 n} \frac{1}{k} \leq K \sup _{k \geq n}\left(k^{1-\alpha} c_{k}\right) \text {. }
$$

Hence

$$
n^{-\alpha} \sum_{k=n}^{2 n} c_{k}=o(1) \quad \text { as } \quad n \rightarrow \infty .
$$

If $\left(c_{n}\right) \in G M\left(\beta^{\#}, r\right)$, then for $\alpha \leq 0$ we get

$$
\begin{array}{r}
n^{1-\alpha} \sum_{k=n}^{\infty}\left|\Delta_{r} c_{k}\right|=n^{1-\alpha} \sum_{s=0}^{\infty} \sum_{k=2^{s} n}^{2^{s+1} n-1}\left|\Delta_{r} c_{k}\right| \leq K n^{1-\alpha} \sum_{s=0}^{\infty} \frac{1}{2^{s} n} \max _{k \geq\left[2^{s} n / c\right]}\left(\sum_{s=k}^{2 k} c_{s}\right) \\
\leq K n^{-\alpha} \max _{k \geq[n / c]}\left(\sum_{s=k}^{2 k} c_{s}\right) \sum_{s=0}^{\infty} \frac{1}{2^{s}} \leq K \max _{k \geq[n / c]}\left(k^{-\alpha} \sum_{s=k}^{2 k} c_{s}\right) .
\end{array}
$$

Thus (2.8) is also valid. 
4.6. Proof of Theorem 7. We prove the theorem for the case when $\phi(x)=g(x)$, only. The case when $\phi(x)=f(x)$ can be proved similarly.

First, using the usual argument

$$
E_{n}\left(\phi, \omega_{\alpha, r}\right)=\inf _{P_{n} \in \Pi_{n}}\left\|\omega_{\alpha, r}\left(\phi-P_{n}\right)\right\| \leq\left\|\omega_{\alpha, r}\left(\phi-S_{n}\right)\right\|=\left\|R_{n+1}\right\|,
$$

where $P_{n}$ is a trigonometric polynomial of degree $n$,

$$
S_{n}=\sum_{k=1}^{n} b_{k} \sin k x
$$

and

$$
R_{n+1}(x)=\sum_{k=n+1}^{\infty} b_{k} \omega_{\alpha, r}(x) \sin k x .
$$

Further, we will show that

$$
\left\|R_{n+1}\right\| \leq K \max _{v \geq n}\left(v^{1-\alpha} \sum_{k=v}^{\infty}\left|\Delta_{r} b_{k}\right|\right) .
$$

Since $R_{n+1}\left(\frac{2 l \pi}{r}\right)=0$, where $l \in \mathbb{Z}$, it suffices to prove (4.1) for $x \in\left(\frac{2 l \pi}{r} ; \frac{2(l+1) \pi}{r}\right)$, where $l=0,1, \ldots,[r / 2]-1$ if $r$ is an even number, and $l=0,1, \ldots,[r / 2]$ if $r$ is an odd number.

First, we show that (4.12) is valid for $x \in\left(\frac{2 l \pi}{r} ; \frac{2 l \pi}{r}+\frac{\pi}{r}\right]$. Let $x \in\left(\frac{2 l \pi}{r} ; \frac{2 l \pi}{r}+\frac{\pi}{r}\right]$ and $j:=\left[\pi /\left(x-\frac{2 l \pi}{r}\right)\right]$. Then, using Lemma 1 and the inequality (4.5), we obtain that for $\alpha \in(0 ; 1]$

$$
\begin{aligned}
&\left|R_{n+1}(x)\right| \leq K\left(j^{-\alpha} \sum_{k=n}^{n+j-1} b_{k}+j^{1-\alpha} \sum_{k=n+j}^{\infty}\left|\Delta_{r} b_{k}\right|\right) \\
& \leq K\left(\max _{n \leq k \leq n+j}\left(k^{1-\alpha} b_{k}\right)+\left(\frac{j}{n+j}\right)^{1-\alpha} \max _{v \geq n+j}\left(v^{1-\alpha} \sum_{k=v}^{\infty}\left|\Delta_{r} b_{k}\right|\right)\right) \\
& \leq K \max _{v \geq n}\left(v^{1-\alpha} \sum_{k=v}^{\infty}\left|\Delta_{r} b_{k}\right|\right) .
\end{aligned}
$$

Now, we prove that (4.12) is valid for $x \in\left[\frac{2 l \pi}{r}+\frac{\pi}{r} ; \frac{2(l+1) \pi}{r}\right)$.

Let $x \in\left[\frac{2 l \pi}{r}+\frac{\pi}{r} ; \frac{2(l+1) \pi}{r}\right)$ and $i=\left[\pi /\left(\frac{2(l+1) \pi}{r}-x\right)\right]$. Applying Lemma 1 and the inequality (4.10) we get, for $\alpha \in(0 ; 1]$,

$$
\left|R_{n+1}(x)\right| \leq K\left(i^{-\alpha} \sum_{k=n}^{n+i-1} b_{k}+i^{1-\alpha} \sum_{k=n+i}^{\infty}\left|\Delta_{r} b_{k}\right|\right) \leq K \max _{v \geq n}\left(v^{1-\alpha} \sum_{k=v}^{\infty}\left|\Delta_{r} b_{k}\right|\right) .
$$

Collecting the above estimates, we arrive at (4.12). The proof is now complete.

\section{References}

[1] T. W. Chaundy, A. E. Jolliffe, The uniform convergence of a certain class of trigonometrical series, Proc. London Math. Soc. 15 (1916), 214-216. 
[2] M. Dyachenko, S. Tikhonov, Integrability and continuity of functions represented by trigonometric series: coefficients criteria, Studia Math. 193 (2009), 285-306.

[3] R. J. Le, S. P. Zhou, A new condition for the uniform convergence of certain trigonometric series, Acta Math. Hungar. 108 (2005), 161-169.

[4] L. Leindler, On the uniform convergence and boundedness of a certain class of sine series, Anal. Math. 27 (2001), 279-285.

[5] L. Leindler, A new class of numerical sequences and its applications to sine and cosine series, Anal. Math. 28 (2002), 279-286.

[6] L. Leindler, A new extension of monotone sequence and its application, JIPAM. J. Inequal. Pure Appl. Math. 7 (2006), Art. 39, 7 pp.

[7] L. Leindler, Necessary and sufficient conditions for uniform convergence and boundedness of a general class of sine series, Aust. J. Math. Anal. Appl. 4 (2007), no. 1, Art. 10.

[8] L. Leindler, Integrability conditions pertaining to Orlicz space, JIPAM. J. Inequal. Pure and Appl. Math. 8 (2007), Art. 38, 6 pp.

[9] J. R. Nurcombe, On the uniform convergence of sine series with quasimonotone coeffcients, J. Math. Anal. Appl. 166 (1992), 577-581.

[10] B. Szal, A note on the uniform convergence and boundedness a generalized class of sine series, Comment. Math. 48 (2008), 85-94.

[11] B. Szal, On L-convergence of trigonometric series, J. Math. Anal. Appl. 373 (2011), 449-463.

[12] B. Szal, A new class of numerical sequences and its applications to uniform convergence of sine series, Math. Nachr. (in press), available online at http://onlinelibrary.wiley.com/doi/10.1002/mana.200910061/pdf

[13] S. Tikhonov, Trigonometric series with general monotone coefficients, J. Math. Anal. Appl. 326 (2007), 721-735.

[14] S. Tikhonov, On uniform convergence of trigonometric series, Mat. Zametki 81 (2007), 304-310, English transl.: Math. Notes 81 (2007), 268-274.

[15] S. Tikhonov, Best approximation and moduli of smoothness: computation and equivalence theorems, J. Approx. Theory 153 (2008), 19-39.

[16] D. S. Yu, S. P. Zhou, A generalization of monotonicity and applications, Acta Math. Hungar. 115 (2007), 247-267.

[17] D. S. Yu, S. P. Zhou, P. Zhou, Ultimate generalization to monotonicity for uniform convergence of trigonometric series, Sci. China Math. 53 (2010), 1853-1862.

[18] D. S. Yu, S. P. Zhou, P. Zhou, On the relations among best approximation and Fourier coefficients, Sci. China Ser. A 51 (2008), 1883-1894.

[19] A. Zygmund, Trigonometric Series, vol. I, Cambridge Univ. Press, New York, 1959. 